EDITORIAL

\title{
DMDs Are an Essential Issue But Are They the Only Issue in MS Treatment?
}

Can J Neurol Sci. 2013; 40: 276-277

The past two decades have witnessed substantial progress in our understanding of the pathogenesis of multiple sclerosis (MS), an improvement in our ability to diagnose the disease and monitor its course and the emergence of MS as a treatable neurological disease. Nevertheless, the development of effective treatments for MS has been impeded by several characteristics of the disease. Disease modifying drugs (DMDs) are now largely prescribed, but there is some risk that all therapeutic efforts shift to the selection and management of these medications neglecting treatment of the symptoms which in themselves represent the daily challenge for both the patient and his clinician.

In this issue of the Canadian Journal of Neurological Sciences, Mark S. Freedman and a group of neurologists working in Canadian MS clinics have met and put together an opinion paper representing the consensus of the group. ${ }^{1}$ This is an update of the Canadian MS Work Group (CMSWGs) recommendations originally published in 2004. In this paper, the authors address some practical recommendations to assist clinicians in optimizing the use of DMDs in patients with relapsing remitting MS. The work was shared among the members of the CMSWGs: members split in working groups each exploring a problem in the choice of DMDs: how is disease activity assessed? how to assess suboptimal response? how to switch or escalate treatment? The split sessions focused on practical criteria to better define relapses, disability progression and MRI worsening. They were developed independently and classified the clinical level of concern as Low, Medium and High. The group recommends that a change in treatment should be considered in any relapsing remitting multiple sclerosis patient, if one identifies a high level of concern in any domain (relapse, progression or MRI); a medium level of concern in any two domains, or a low level of concern in all three domains. ${ }^{1}$

Despite major advances in understanding the scientific basis for MS, and in diagnosis and treatment, the problem of misdiagnosis persists. Solomon and colleagues conducted a survey of neurologists with special interest in MS. From the survey results, they emphasized that nearly all MS specialists who completed the survey had seen patients in the prior year for whom they thought an MS diagnosis was incorrect. Furthermore, many of the patients with an incorrect MS diagnosis were actually taking MS DMDs! ${ }^{2}$ Therefore, it appeared appropriate as an emergent issue at the beginning of a national consensus of MS to address MS diagnostic criteria. Indeed, a number of diseases such as encephalitis, migraine headaches ${ }^{3}$, Vitamin B12 deficiency $^{4}$, CLIPPER Syndrome ${ }^{5,6}$ and many vasculitis ${ }^{7}$ can have similarities with MS but require different treatments.

We would also have liked to see this group of experts take more attention to consequences of neutralizing antibodies in Interferon treated patients. They recommended to measure neutralizing antibodies but a void is left on how should one be acting on these results. It is probably because of the dollar value of the North American market that the issue is hotly debated in North America and that no consensus has arisen despite the evidence that antibodies inhibit the MRI effect of Interferons. The position of BiogenIdec concerning Tysabri ${ }^{\circledR}$ treated patients developing antibodies, has been clearer and the recommendation to stop the medication put forward from the onset.

The manuscript contains a number of excellent points. Changing the definition of relapse time from 24 hours to 48 hours, is very practical and an excellent choice. ${ }^{1}$ In contrast, with transient ischemic attacks and fever, worsening in demyelinating diseases takes more than 48 hours to peak. Personally, we would even prefer five to seven days as the definition of relapse in MS.

The other fascinating topics, which were discussed, are the evaluation of cognitive function, depression and fatigue in the clinical course. ${ }^{1}$ However, the group has not managed to generate a consensus on how to evaluate and follow this dreadful aspect of MS symptoms. It is interesting that this group takes such a firm position on the emphasis of vitamin D3. This is probably because Canada (north of 49th parallel) has a large part of the country exposed to long nights in the winter. Nevertheless, the problem could be addressed better by public health specialists as hypovitaminosis D is a country-wide problem and should be sought as a risk for higher relapse in MS. ${ }^{8}$

To conclude, we would stress that a comprehensive treatment of MS should, in addition to implementing DMDs, emphasize symptomatic treatments and exercise which are an essential part of MS management and probably amplify the benefit of DMDs.

Saeed Shahbeigi, Joel Oger

University of British Columbia Multiple Sclerosis Clinic and Neuro-Immunology Laboratory UBC Hosptial, Vancouver, British Columbia, Canada 


\section{REFERENCES}

1. Freedman MS, Selchen D, Arnold DL, et al. Treatment optimization in MS: Canadian MS Working Group updated recommendations. Can J Neurol Sci. 2013;40(3):307-23.

2. Solomon AJ, Klein EP, Bourdette D. "Undiagnosing" multiple sclerosis: the challenge of misdiagnosis in MS. Neurology. 2012; 78:1986-91.

3. Rolak LA, Fleming JO. The differential diagnosis of multiple sclerosis. Neurologist. 2007;13:57-72.

4. Najafi MR, Shaygannajad V, Mirpourian M, Gholamrezaei A. Vitamin B12 deficiency and multiple sclerosis; is there any association? Int J Prev Med. 2012;Apr 3(4):286-9.
5. Jones JL, Dean AF, Antoun N, Scoffinqs DJ, Burnet NG, Coles AJ. 'Radiologically compatible CLIPPERS' may conceal a number of pathologies. Brain. 2011;34:1-3.

6. Kastrup O, Van De Nes J, Gasser T, Keyvani K. Three cases of CLIPPERS: a serial clinical, laboratory and MRI follow-up study. J Neurol. 2011;258:2140-6.

7. Scolding N. The differential diagnosis of MS. J Neurol Neurosurg Psychiatry. 2001;71 Suppl II:ii9-ii15.

8. Runia TF, Hop WCJ, de Rijke YB, Buljevac D, Hintzen RQ. Lower serum vitamin $\mathrm{D}$ levels are associated with a higher relapse risk in multiple sclerosis. Neurology. 2012;79:261-6. 